\title{
A Stable Double-Tilt Heating Capability for Precision Atomic-Level Imaging of Catalysts at Elevated Temperatures
}

\author{
Lawrence F. Allard,* Wilbur C. Bigelow, ${ }^{* *}$ Douglas A. Blom*** and Jingyue (Jimmy) Liu**** \\ * Materials Science and Technology Division, Oak Ridge National Laboratory, TN 37831 \\ **Department of Materials Science \& Engineering, University of Michigan, Ann Arbor, MI 48104 \\ *** Electron Microscopy Center, Univ. of South Carolina, 715 Sumter St., Columbia, SC 29208 \\ ****Center for Nanoscience, Department of Physics \& Astronomy, Department of Chemistry \& \\ Biochemistry, University of Missouri-St. Louis, MO 63121
}

We have recently described a novel in situ heating capability employing MEMS-based heater devices (Aduro ${ }^{\mathrm{TM}}$, Protochips Inc., Raleigh, NC) that allows full sub-Ångström resolution on catalytic materials via aberration-corrected high-angle annular dark-field imaging (HAADF) in microscopes such as the JEOL 2200FS STEM/TEM [1]. That technology presently only permits the single-tilt operation of the microscope's goniometer to be used for appropriate alignment of crystal zone axes. Since HAADF imaging is critically dependent on precise alignment of the electron beam with atomic columns in the sample, we have fabricated a double-tilt specimen holder that provides the required orthogonal tilt axis $\left( \pm 6^{\circ}\right)$ while allowing heating to $>1000^{\circ} \mathrm{C}$ and maintaining the single-atom imaging capability. The new capability improves our ability to study e.g. growth and behavior of catalytic nanoparticles on nanoscale single-crystal supports, and the dynamic behavior of interface structures at elevated temperatures.

The double-tilt specimen holder for Aduro devices is shown in Fig. 1a. The Aduro device is held on a 'tilt table' by the electrical leads, which clamp onto gold electrodes on the device near the axis of the tilt-table bearing supports. The tilt action is controlled by pushing or pulling on the end of the tilt table using a pushrod attached to a wedge that contacts a thin shaft at the end of the table, as seen in Fig. 1b. The shaft is held firmly against the tilt wedge by a thin phosphor bronze shim (arrow). Gearing in a motor drive in the handle of the holder is set to move the full tilt from $0^{\circ}$ to $\pm 6^{\circ}$ in about 2 minutes. This tilt speed allows precise control of the tilt action, and eucentricity is maintained by the design to permit easy setting of the internal tilt to obtain the imaging geometry required. Examples of the utility of the holder include the study of coalescence and growth of $\mathrm{Pd}$ and $\mathrm{PdZn}$ nanoparticles on $\mathrm{ZnO}$ nanoribbons shown here [2].

Figure 2 shows use of the double-tilting operation to set a $\mathrm{ZnO}$ nanoribbon into a precise $<11-20>$ zone axis orientation. Figure 3 shows the benefit of achieving perfect alignment of the nanoribbon with the zone axis, as the atomic columns of $\mathrm{Zn}$ are well resolved in comparison with the image of the same area at an off-axis tilt of about $2^{\circ}$. Figure 4 shows a PdZn nanoparticle, formed after heating at $700^{\circ} \mathrm{C}$ for 30 min., on the perfectly oriented $\mathrm{ZnO}$ support, with $\mathrm{Zn}$ columns well resolved and the epitaxy between the $\mathrm{PdZn}$ nanoparticle and the $\mathrm{ZnO}$ support crystal clearly evident. Application of the double-tilt heater to a study of the structure of the "M1" phase of a MoVNbTeO complex oxidation catalyst at catalytically relevant temperatures will also be discussed [3].

\section{References}

[1] L. F. Allard, et al., Micros. Res. \& Tech., 72(3) 208-215 (2008). 
[2] J. Liu and L. F. Allard, $21^{\text {st }}$ NACS meeting proceedings, QD50 (2009).

[3] Microscopy research at the Oak Ridge National Laboratory's High Temperature Materials Laboratory was sponsored by the U. S. Department of Energy, Office of Energy Efficiency and Renewable Energy, Vehicle Technologies Program.

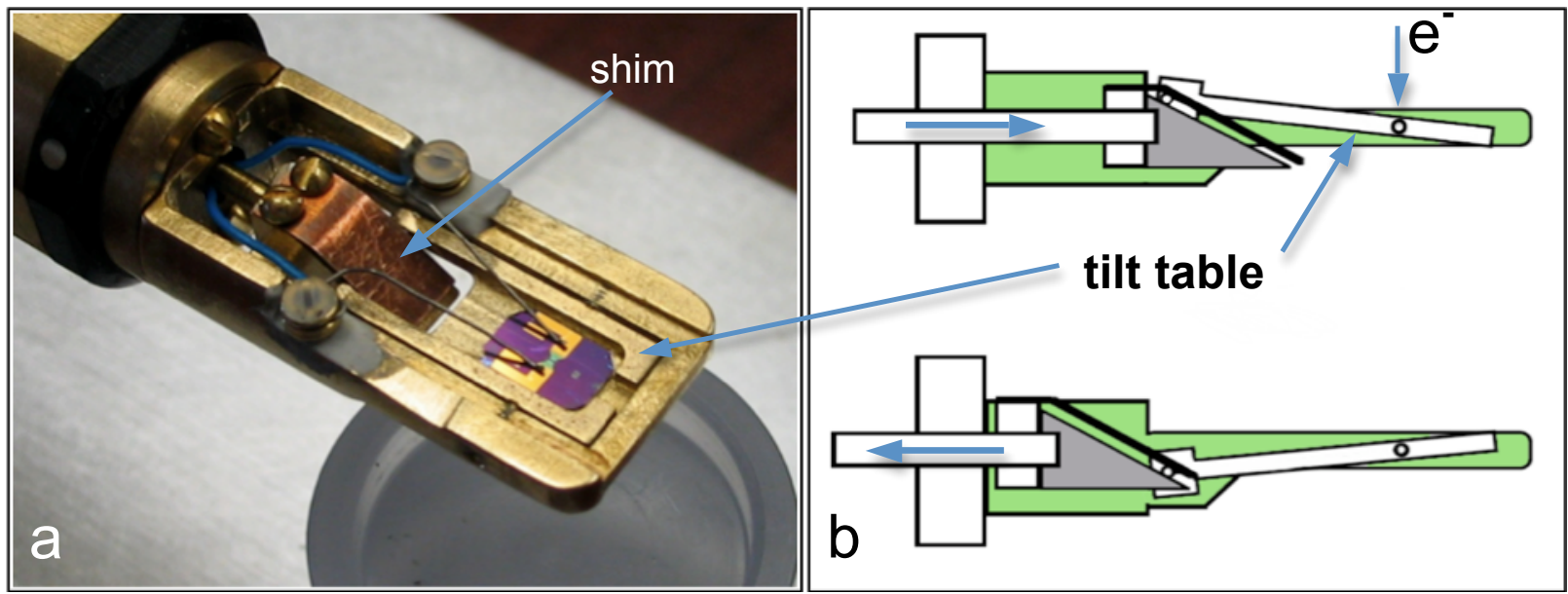

FIG. 1. a) View of the tip of the double-tilt heating holder, showing Aduro chip in place, held by electrical clip leads; b) cross-section schematic showing 2 positions of tilting action.

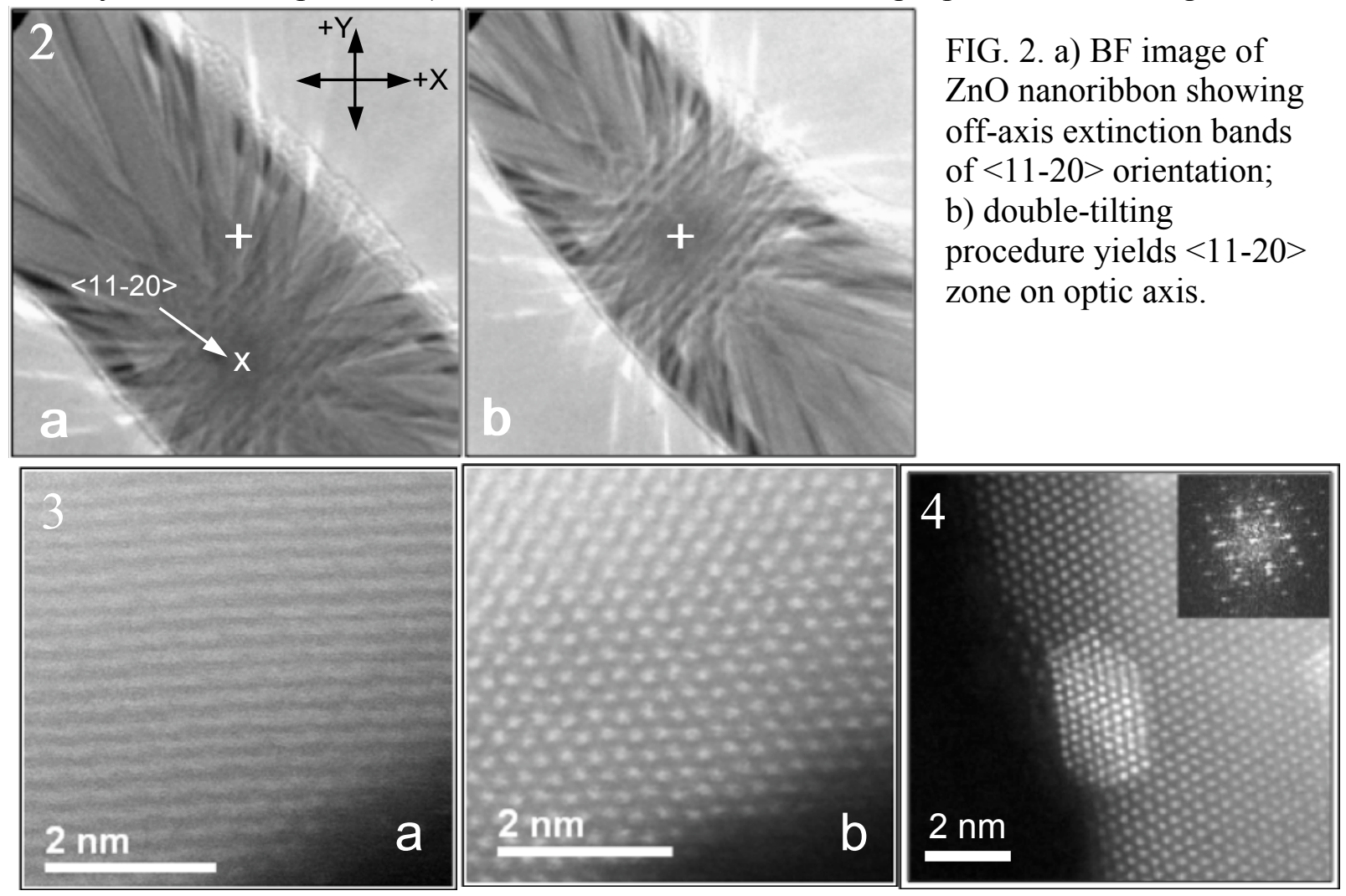

FIG. 3. a) HAADF image at $15 \mathrm{Mx}$ original magnification, with $\mathrm{ZnO}$ nanoribbon $\sim 2^{\circ}$ off axis; b) same area with nano-ribbon oriented on zone axis.

FIG. 4. PdZn nanoparticle on $\mathrm{ZnO}$ nanoribbon, showing epitactical orientation on the support, clearly observed with perfect orientation of the nanoribbon with the electron beam. 